\title{
Relationship between the Fundamental Constants of Physics Obtained from the Uncertainty Principle for Energy and Time
}

\author{
Stanisław Olszewski \\ Institute of Physical Chemistry, Polish Academy of Sciences Kasprzaka, Warsaw, Poland \\ Email: olsz@ichf.edu.pl
}

Received 27 January 2015; accepted 15 April 2015; published 16 April 2015

Copyright (C) 2015 by author and Scientific Research Publishing Inc.

This work is licensed under the Creative Commons Attribution International License (CC BY).

http://creativecommons.org/licenses/by/4.0/

(c) (1) Open Access

\section{Abstract}

An attempt is done to calculate the value of the elementary electron charge from its relation to the Planck constant and the speed of light. This relation is obtained, in the first step, from the Pauli analysis of the strength of the electric field associated with an elementary emission process of energy. In the next step, the uncertainty principle is applied to both the emission time and energy. The theoretical result for $e$ is roughly close to the experimental value of the electron charge.

\section{Keywords}

Fundamental Constants of Physics, Uncertainty Principle for Energy and Time

\section{Introduction}

As soon as the atomic theory of matter occured to be a right idea, there arose a tendency to describe the physical properties of matter with the aid of a possibly low number of the elementary notions concerning the atoms and their structure. A further step in this direction has been provided by the quantum theory. In effect numerous properties of the atomic world could be represented in terms of the so-called fundamental constants of physics which are evidently few in their number. Perhaps the most widely used constants became $e, m, h$ and $c$, which are respectively the electron charge and electron mass, the elementary action called the Planck constant and the speed of light.

Simultaneously a mutual reference between the constants mentioned above seemed to be not so much evident. The aim of the present paper is to demonstrate that, in fact, a reference between $e, h$ and $c$ can be supplied in effect of i) an elementary analysis of the forces entering the emission process of the electron energy, ii) an application of the uncertainty principle for energy and time which couples the parameters considered in i).

How to cite this paper: Olszewski, S. (2015) Relationship between the Fundamental Constants of Physics Obtained from the Uncertainty Principle for Energy and Time. Journal of Modern Physics, 6, 622-626.

http://dx.doi.org/10.4236/jmp.2015.65067 
The energy-time aspect of the uncertainty principle has been presented originally by Heisenberg [1] (see also e.g. [2]) in the formula

$$
\Delta E \Delta t>\hbar
$$

where $\Delta E$ is the energy change in a quantum process the duration of which is $\Delta t$. But (1) has been objected on many occasions [3]-[5] and in fact numerous textbooks on quantum mechanics neglect (1) at all; see e.g. [6]. However, a modified approach to the coupling between $\Delta E$ and $\Delta t$ is also possible [7]-[9]. This gives instead of (1) a relation

$$
2 m c^{2} \Delta E(\Delta t)^{2}>\hbar^{2}
$$

which on many occasions can be replaced by an approximate equation

$$
2 m c^{2} \Delta E(\Delta t)^{2} \approx \hbar^{2} .
$$

The formula (2a) allowed us to approach several problems of the elementary quantum theory, for example the spectrum of the Bohr hydrogen atom [10] and the spin mechanism [11] [12]. A check of (2) has been done in [13]. It gives the formula

$$
2 m c^{2} \Delta E(\Delta t)^{2}>\frac{8}{n} \hbar^{2}
$$

where $\Delta E$ is the energy of transitions between the Bohr quantum levels $n$ and $n+1$ and $\Delta t$ is the time of transition estimated in [13]. Evidently for $n$ close to 10 the formula (2b) approaches (2a) and for $n \gg 8$ there is satisfied the relation (2). In the present paper the aim of (2a) is to put a bridge between $e, h$ and $c$.

One of the notions useful to this purpose is a minimal distance between two particles having the same mass $m$. This is

$$
\Delta x=\frac{\hbar}{m c} .
$$

Equation (3) has been derived also on the basis of the formula (2a); see [10]. Before (2a) and (3) are applied, we refer to the Pauli analysis of the radiation emission process.

\section{Pauli Analysis of the Electric Field Involved in the Radiation Emission Process [14]}

Pauli's idea was to consider the strength of the electric field $\boldsymbol{E}$ connected with an oscillator having a definite frequency $v$. The change of the number of quanta of the oscillator connected with the emission process is unknown. The average frequency $v$ of the emitted light let be referred to the interval of time $\Delta t$ by the relation

$$
v \sim \frac{1}{\Delta t}
$$

In effect the intensity square of the electric field $\boldsymbol{E}$ is coupled with $\Delta t$ by the formula

$$
\boldsymbol{E}^{2} \sim \frac{v^{3}}{c^{3}} \frac{h v}{2} \sim \frac{h c}{(c \Delta t)^{4}} .
$$

In order to derive (4a) two formulae for the momentum change $\Delta p$ within the time interval $\Delta t$ can be considered. These are

$$
\Delta p \Delta t>\frac{h}{v-v^{\prime}}
$$

where $\Delta p$ is the momentum change of an arbitrary body, and 


$$
\Delta p \Delta t>\frac{e^{2}}{c^{3}}\left(v^{\prime}-v\right)
$$

where $\Delta p$ is the momentum change due to the radiation process. An effect of multiplication of (5) and (6) is

$$
(\Delta p \Delta t)^{2} \sim \frac{e^{2} h}{c^{3}}
$$

or

$$
\Delta p \Delta t \sim \frac{e h^{1 / 2}}{c^{3 / 2}} .
$$

On the other hand the change $\Delta p$ in effect of a change of the electric field $\Delta \boldsymbol{E}$ in time $\Delta t$ leads to relation

$$
e|\Delta \boldsymbol{E}| \Delta t>\Delta p
$$

so

$$
e|\Delta \boldsymbol{E}|(\Delta t)^{2}>\Delta p \Delta t \sim \frac{e h^{1 / 2}}{c^{3 / 2}} .
$$

Therefore a final formula gives

$$
|\Delta \boldsymbol{E}|^{2}>\frac{h}{c^{3}(\Delta t)^{4}}=\frac{h c}{(c \Delta t)^{4}} .
$$

According to Pauli the square root of $|\Delta \boldsymbol{E}|^{2}$ exceeds the square root of the expression presented on the right of (9); see also (4a).

However, for simplicity, let us assume that this excess is small and the change of the field intensity in the emission is

$$
|\Delta \boldsymbol{E}| \sim \frac{(h c)^{1 / 2}}{(c \Delta t)^{2}} .
$$

In result the force acting on the electron particle becomes

$$
e|\Delta \boldsymbol{E}| \sim e \frac{(h c)^{1 / 2}}{(c \Delta t)^{2}} .
$$

We assume that the force given in (11) is acting along an elementary (minimal) space interval $\Delta x$ presented in (3). In effect the change $\Delta E$ of the electron energy obtained along the distance $\Delta x$ amounts to

$$
\Delta E=\Delta x e|\Delta \boldsymbol{E}| \sim \frac{\hbar}{m c} e \frac{(h c)^{1 / 2}}{(c \Delta t)^{2}} .
$$

\section{Energy $\Delta E$ of (12) Applied in the Uncertainty Formula (2a) Gives an Equation for $e$}

As a final step we substitute the energy change (12) to the uncertainty formula (2a). This gives an approximate equation

$$
2 m c^{2} \Delta E(\Delta t)^{2} \approx 2 m c^{2} \frac{\hbar}{m c} e \frac{(h c)^{1 / 2}}{(c \Delta t)^{2}}(\Delta t)^{2} \approx \hbar^{2}
$$


which can be simplified to the relation

$$
\frac{h}{\pi}\left(\frac{h}{c}\right)^{1 / 2} e \approx \frac{h^{2}}{4 \pi^{2}}
$$

or

$$
e \approx \frac{(h c)^{1 / 2}}{4 \pi}
$$

coupling the constants $h, c$, and $e$. A substitution of

$$
h=6.62 \times 10^{-27} \mathrm{erg} \cdot \mathrm{sec}
$$

and

$$
c=3 \times 10^{10} \mathrm{~cm} \cdot \mathrm{sec}^{-1}
$$

into (15) gives

$$
e=1.12 \times 10^{-9} \text { esu. }
$$

The well-known value of the measured $e$ is

$$
e=0.48 \times 10^{-9} \text { esu. }
$$

The difference between (18) and (18a) is about 50 percent of (18). In some earlier papers (see [15]-[17] and also [18] instead of (3) the Compton length

$$
\Delta x^{\text {Compton }}=\frac{h}{m c}
$$

is proposed which is larger than $\Delta x$ in (3) by the factor of $2 \pi$. This would imply that $e$ in (18) should be smaller by the factor of $2 \pi$, so

$$
e^{\text {Compton }}=\frac{1.12}{2 \pi} \times 10^{-9} \text { esu }=0.18 \times 10^{-9} \text { esu. }
$$

An approximate character of the formalism applied in the present paper is evident.

\section{Summary}

The electron charge $e$ has been calculated from the Planck constant $h$ and the speed of light $c$. This has been done on the basis of i) the Pauli expression for the emission strength of the electric field, ii) the uncertainty principle for energy and time. The emitted energy is obtained as a product of the force of the electric field and the elementary distance $\Delta x$ [see (3)] derived from the principle mentioned in ii); see [10].

In case the factor of $1 / 2$ introduced by Pauli as a multiplier of $h v$ [see (4a)] is taken explicitly into account, the result for $e$ in (18) is transformed into

$$
e=\frac{1.12}{2^{1 / 2}} \times 10^{-9} \text { esu }=0.79 \times 10^{-9} \text { esu. }
$$

This number is different by less than 40 percent of its value from the experimental $e$ represented in (18a).

The particle mass does not interfere in the equations of the paper. This implies that the absolute value of $e$ can be the same for electrons and protons.

\section{References}

[1] Heisenberg, W. (1927) Zeitschrift fuer Physik, 43, 172. http://dx.doi.org/10.1007/BF01397280 
[2] Schiff, L.I. (1968) Quantum Mechanics. 3rd Edition, McGraw-Hill, New York.

[3] Schommers, W. (1989) Space-Time and Quantum Phenomena. In: Schommers, W., Ed., Quantum Theory and Pictures of Reality, Springer, Berlin. http://dx.doi.org/10.1007/978-3-642-95570-9_5

[4] Allcock, G.R. (1969) Annals of Physics, 53, 253. http://dx.doi.org/10.1016/0003-4916(69)90251-6

[5] Bunge, M. (1970) Canadian Journal of Physics, 48, 1410. http://dx.doi.org/10.1139/p70-172

[6] Isaacs, A. (1990) Concise Dictionary of Physics. Oxford University Press, Oxford.

[7] Olszewski, S. (2011) Journal of Modern Physics, 2, 1305. http://dx.doi.org/10.4236/jmp.2011.211161

[8] Olszewski, S. (2012) Journal of Modern Physics, 3, 217. http://dx.doi.org/10.4236/jmp.2012.33030

[9] Olszewski, S. (2012) Quantum Matter, 1, 127. http://dx.doi.org/10.1166/qm.2012.1010

[10] Olszewski, S. (2014) Journal of Modern Physics, 5, 1264. http://dx.doi.org/10.4236/jmp.2014.514127

[11] Olszewski, S. (2014) Journal of Modern Physics, 5, 2022-2029. http://dx.doi.org/10.4236/jmp.2014.518198

[12] Olszewski, S. (2014) Journal of Modern Physics, 5, 2030-2040. http://dx.doi.org/10.4236/jmp.2014.518199

[13] Olszewski, S. (2012) Quantum Matter, 1, 59-62. http://dx.doi.org/10.1166/qm.2012.1006

[14] Pauli, W. (1933) Die allgemeine Prinzipien der Wellenmechanick. In: Geiger, H. and Scheel, K., Eds., Handbuch der Physik, Vol. 24, Part 1, Springer, Berlin.

[15] Ruark, A.E. (1928) Proceedings of the National Academy of Sciences of the United States of America, 14, 322-328. http://dx.doi.org/10.1073/pnas.14.4.322

[16] Flint, H.E. (1928) Proceedings of the Royal Society A: Mathematical, Physical and Engineering Sciences, 117, 630637. http://dx.doi.org/10.1098/rspa.1928.0025

[17] Flint, H.E. and Richardson, O.W. (1928) Proceedings of the Royal Society A: Mathematical, Physical and Engineering Sciences, 117, 637-649. http://dx.doi.org/10.1098/rspa.1928.0026

[18] Jammer, M. (1966) The Conceptual Development of Quantum Mechanics. McGraw-Hill, New York. 